\title{
Linealización del Campo de Medida del Manómetro de Bourdon con Engranajes No Circulares
}

\author{
Bourdon Gage Span Linearization with Non-Circular Gears
}

\author{
A. Manotas-Cantillo , J. Silva-Gutiérrez iD , J. Roldán-Mckinley iD
}

\begin{abstract}
Resumen- En este trabajo se documentó la síntesis de una conexión de engranajes no circulares que es integrada a un manómetro de Bourdon. El uso de la conexión de engranajes tiene como objetivo compensar el comportamiento no lineal de la deformación del tubo de Bourdon. La relación de entrada-salida de la conexión fue deducida mediante el análisis de un mecanismo de cuatro barras equivalente. La deformación del tubo de Bourdon se modela como un eslabón rígido y su ángulo de posición se consideró como la variable de entrada. El ángulo descrito por la aguja indicadora de la presión solidaria al eslabón se establece como el ángulo de salida. Los ángulos de salida ideales deberían permitir un movimiento lineal de la aguja indicadora mientras se mantienen divisiones de igual amplitud a lo largo de la escala. La relación de entrada-salida de la conexión, los ángulos de salida y entrada, junto con la distancia entre centros permitió la síntesis de la porción de la curva de paso sobre la cual se debería hacer el diseño de los dientes. La formulación matemática fue programada en GNU Octave para la generación de las curvas de ángulo de salida, relación de engranajes y curvas de paso. Se contrarrestó la no linealidad del manómetro de Bourdon mediante la integración de elementos mecánicos de perfil no circular (no lineales). Se logró linealizar la escala de medición para una resolución uniforme en todo el campo de medida, resultados verificados gráficamente mediante la comparación de las curvas de paso contra el movimiento de los elementos dentro de un modelo CAD generado en SolidWorks. Lo anterior permite tener una resolución uniforme a lo largo del campo de medida.
\end{abstract}

Palabras claves - Diseño de mecanismos y máquinas; engranajes no circulares; instrumentación industrial; manómetro de Bourdon; perfil de paso; síntesis de mecanismos.

Este manuscrito fue enviado el 04 de febrero de 2020 y aceptado el 20 de junio de 2020. Este artículo fue apoyado por Vicerrectoría de Investigaciones, Extensión y Proyección Social de la Universidad del Atlántico (Puerto Colombia, Colombia).

A. Manotas-Cantillo es Ingeniero Mecánico egresado de la Universidad del Atlántico (Puerto Colombia, Colombia). (e-mail: afmanotas@mail.uniatlantico.edu.co).

J. Silva-Gutierrez es Ingeniero Mecánico egresado de la Universidad del Atlántico (Puerto Colombia, Colombia) (e-mail: jesilva@mail.uniatlantico.edu.co).

J. Roldán-Mckinley trabaja actualmente en el Programa de Ingeniería Mecánica (DIMER, grupo de investigación) en la Universidad del Atlántico (Puerto Colombia, Colombia) (e-mail: javierroldan@mail.uniatlantico.edu.co). Autor de correspondencia
Abstract - It is reported the synthesis of a non-circular gear connection portion to be used in a Bourdon pressure gauge. The purpose of the gear connection is compensating the bellows' deformation non-linearity. The connection Input-Output (I/O) gear ratio was deduced by identifying and analyzing a four-bar linkage. The bellows deformation was represented as a rigid element and its position angle was set as the mechanism input variable. The angle described by the pointer element (pressure indicator) is established as the connection output angle. The ideal output angles should allow a linear motion of the pointer while keeping same amplitude divisions along the scale. The I/O gear ratio, the input and output angles, in addition to the center distance allowed the primitive curve portion synthesis along which the teeth would be designed. The mathematical formulation was programmed in GNU Octave in order to visualize the output angles, I/O ratio, and the non-circular gear pitch curves. The nonlinearity of the Bourdon manometer was counteracted by the integration of non-circular (non-linear) profile mechanical elements. Measurement scale was linearized for uniform resolution across the entire measurement range. Findings were validated by comparing the pitch curves against the motion of a CAD model generated in SolidWorks. Thus, an uniform resolution along the measuring span was achieved.

Index Terms - Design of mechanisms and machines; noncircular gears; industrial instrumentation; Bourdon gage manometer; pitch curve; mechanism synthesis.

\section{INTRODUCCIÓN}

$\mathrm{D}$ ESDE la invención del manómetro tipo Bourdon se ha usado como uno de los instrumentos mecánicos de medición de presión más utilizado en la industria. Se emplea como instrumento de control en procesos químicos, instalaciones hidráulicas, neumáticas, HVAC, industria petroquímica, farmacéutica y diversas aplicaciones industriales. Esto se debe a su bajo costo y simplicidad al no requerir una fuente de energía externa, en contraste con los transductores electrónicos actuales. $\mathrm{Su}$ movimiento de naturaleza mecánica se transmite por la acción de un mecanismo interno de cuatro elementos impulsado por la deformación elástica del tubo ante la presión de entrada [1]. El valor de la presión es indicado en el dial del instrumento mediante una aguja conectada al elemento de salida de movimiento del mecanismo, una conexión de engranajes 
rectos circulares se encarga de la amplificación del movimiento y la indicación de la presión medida [2].

De otra parte, los engranes no circulares han resultado ser una buena alternativa a los engranajes circulares comunes en aplicaciones en las cuales no hay linealidades en la transmisión del movimiento, por ese motivo se propone utilizarlo en el mecanismo interno del manómetro tipo Bourdon. Varios autores destacan una particular aplicación de estos elementos de transmisión en mecanismos usados para la generación de funciones [3], alcanzando inclusive, aplicaciones industriales como el caso reportado por Gobler en donde fueron usados engranes no circulares en imprentas [4]. Más recientemente, Dooner [5] desarrolló un mecanismo de 8 barras con engranes no circulares para mejorar el sistema de dirección de un automóvil, además mostró mediante el uso de engranes no circulares la posibilidad de reducir las fluctuaciones de torque y velocidad en ejes [6]. Otros estudios se han enfocado en la síntesis de engranes no circulares como generador de funciones para desempeño de tareas cinemáticas, mostrando distintas trayectorias que se podrían obtener con varias curvas de paso, con estudios de naturaleza teórica basados en método gráfico, analítico o asistido por computador. Sin embargo, los reportes encontrados acerca de los engranajes no circulares no contemplan una integración de éstos con el manómetro de Bourdon; instrumento que desde su invención no ha variado sus elementos constitutivos, ni alterado su principio mecánico de funcionamiento.

Este artículo presenta la integración de engranajes no circulares en el mecanismo de transmisión de movimiento del manómetro tipo Bourdon, para lograr una regulación de la medida final del instrumento. Inicialmente se generó un modelo CAD en SolidWorks del manómetro de Bourdon utilizado como caso de estudio. Posteriormente se realizan una serie de simulaciones a cada unidad de presión de trabajo del manómetro por medio del método de elementos finitos; el resultado de estas simulaciones permitirá establecer la trayectoria descrita por la punta del tubo de Bourdon en todo el campo de medida. La trayectoria trazada por la deformación del tubo de Bourdon será modelada mediante la ecuación general de la circunferencia, caracterizando el elemento como un eslabón equivalente. El eslabón equivalente constituirá así un mecanismo de cadena cerrada, al cual se le realizará un análisis cinemático directo. Tal análisis establecerá las ecuaciones que relacionan la entrada del manómetro (presión de trabajo) con la salida de la aguja indicadora, dichas ecuaciones se programarán en Octave para facilitar el análisis a lo largo del rango de trabajo del instrumento. Esta programación también permitirá corroborar los datos obtenidos de la simulación de elementos finitos y comprobar su veracidad.

Con base en los ángulos de posición para los elementos de entrada y salida del manómetro de Bourdon, se procede a establecer el perfil de paso de los engranajes no circulares con la ayuda del método "Path Planning", con el cual es posible determinar las distintas relaciones de transmisión necesarias para lograr regular el campo de medida del manómetro de Bourdon. El perfil de paso obtenido es simulado en
SolidWorks con el fin de apreciar tanto el movimiento de la aguja como su nueva amplitud de trabajo. Esta simulación permitirá validar el efecto de los perfiles de paso no circulares en el mecanismo del manómetro de Bourdon.

\section{GENERALIDADES DEL MANÓMETRO DE BOURDON}

Los manómetros como instrumentos de medición fueron mencionados por primera vez por el físico alemán Otto Von Guericke en el siglo XV, sin embargo, el primer modelo conocido fue empleado por el naturalista y geólogo suizo Horace Bénédict de Saussure a mediados del siglo XVI. Fue tanto el auge del manómetro y con la rápida expansión del sector industrial, que después de dos siglos de uso del barómetro (el cual se construía a partir de columnas de vidrio con mercurio) se evidenció la necesidad de cambiar drásticamente la manera en que se medía la presión, debido a la dificultad para medir presiones de más de 1 bar, transporte del instrumento y pocos campos de aplicación.

A pesar de que se plantearon diversas soluciones para corregir los problemas inherentes del barómetro y expandir los campos de aplicación, éstas fueron descartadas casi inmediatamente por su impacto económico y operativo, no era conveniente construir columnas de hierro de gran altura y reemplazar el fluido de trabajo por ácido sulfúrico coloreado por lo peligroso de su transporte y la constante re-calibración por la volatilidad del fluido. Sin embargo, en 1849 apareció el primer modelo del conocido manómetro de Bourdon, recibiendo su nombre en honor a su inventor el ingeniero Eugène Bourdon. Este novedoso instrumento de medición de presión, cuyo principio de funcionamiento difería al empleado con los manómetros de mercurio, fue ampliamente utilizado en el campo de las máquinas de vapor por su tamaño y practicidad midiendo altas presiones.

El auge de las calderas de vapor creó la necesidad de controlar fluidos con presiones variables a través de válvulas, lo que ocasionó que el manómetro de Bourdon tuviera mejoras sustanciales en su principio de funcionamiento y la exactitud en la medición. Gracias a esta mejora continua y la constante necesidad de la aplicación en otros campos industriales, aparecieron diversos tipos de manómetros con distintas dimensiones como los manómetros con tubos helicoidales y en espiral [7]. Generalmente, la selección de una configuración de tubo de Bourdon depende de la aplicación, los tubos de Bourdon con forma espiral son utilizados en aplicaciones donde se miden bajas presiones debido que su deformación en la punta es mucho mayor. Los manómetros con tubo helicoidal. Por otro lado, son útiles a la hora de evitar el uso de mecanismos transmisores de movimiento y así lograr construcciones más pequeñas [8].

Aunque el manómetro de Bourdon se haya inventado hace más de 150 años, sigue vigente debido a su simplicidad, control económico de la presión y medición rápida de la misma. Aunque es conocida la tendencia no lineal a la deformación de la punta del tubo de Bourdon, hasta el momento no se ha aplicado una solución definitiva al control de este fenómeno, los fabricantes optan por limitar el uso de sus manómetros sólo en los rangos donde la linealidad es más 
apreciable, agregando elementos linealizantes que reducen el error en la medición como los resortes planos en espiral, que se encargan de reducir la histéresis en la medida; esto implica un sobre diseño en los manómetros para cumplir los requerimientos de calidad exigidos y que podría reducirse con el uso de engranajes no circulares que se proponen en este artículo.

\section{A. Principio de Funcionamiento}

El principio de funcionamiento del manómetro de Bourdon se basa en la deformación de un tubo metálico con sección transversal, generalmente, ovalada o elíptica plana, accionado por una presión de entrada generada por el fluido de trabajo. Dicha deformación se produce por la tendencia del tubo con sección transversal elíptica a volverse circular, y como consecuencia genera un movimiento en la sección cerrada del tubo de Bourdon, llamado "tip", y es transmitida a través de un mecanismo de barras a un par de engranajes circulares. Esta transmisión de movimiento es finalmente indicada en el dial del instrumento como la presión del sistema. Básicamente se puede resumir el manómetro de Bourdon en 3 partes fundamentales: un elemento de medición flexible (tubo de Bourdon), un mecanismo de transmisión de movimiento y un dial indicador, la Fig. 1 especifica los componentes de un manómetro de Bourdon.

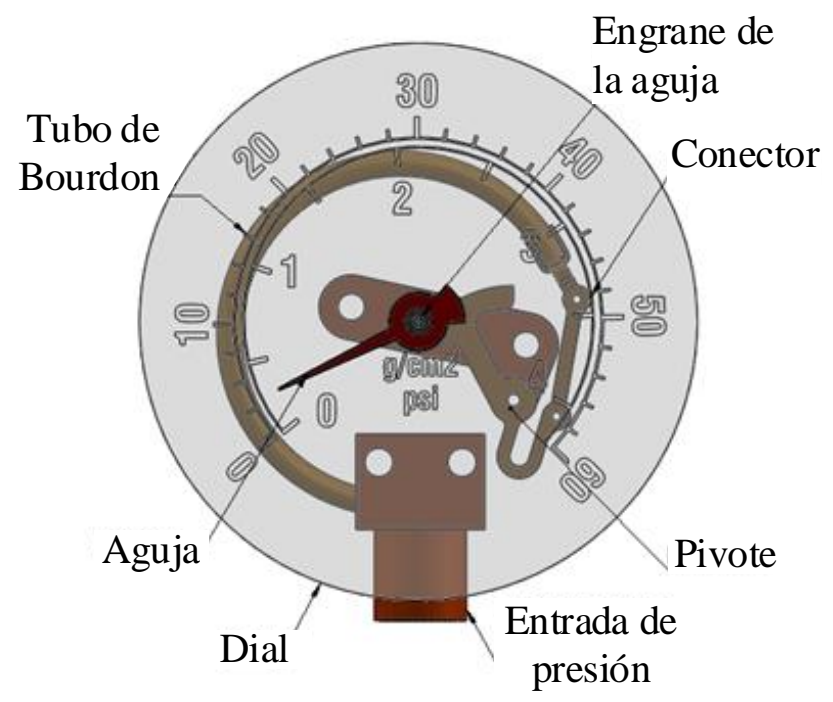

Fig. 1. Esquema de las partes del manómetro de Bourdon.

Las curvas de presión-desplazamiento mostradas en la Fig. 2 muestran la relación típica entre las secciones de tubo de Bourdon más comunes. Dependiendo del tipo de manufactura de estas secciones transversales, se obtendrá una respuesta más o menos lineal. Generalmente, secciones circulares describen curvas convexas, mientras que las secciones ovaladas planas tenderán a describir curvas cóncavas.

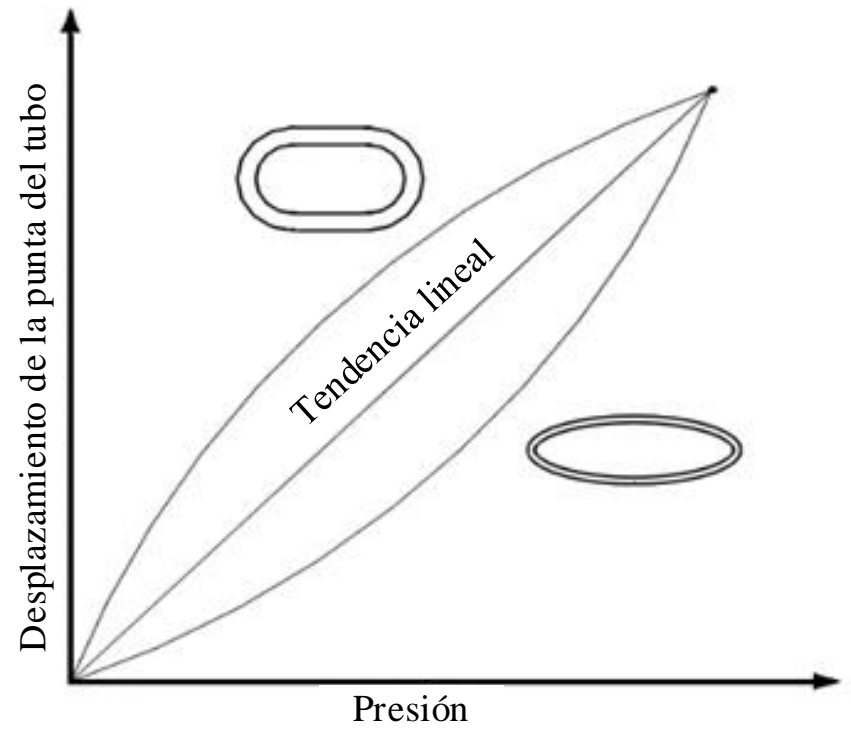

Fig. 2. Curva de presión vs. Desplazamiento con diferentes secciones transversales.

\section{B. Tubo de Bourdon}

El fuelle o tubo de Bourdon es el principal elemento constitutivo del manómetro, debido que es el responsable del movimiento del mecanismo interno del instrumento. Esta pieza se construye a partir de un tubo de sección transversal circular que se aplana hasta volverlo elíptico, y luego se conforma de manera circular o helicoidal. El diseño de un tubo de Bourdon depende principalmente de los rangos de presiones que se vayan a medir o el tipo de fluido de trabajo. En los casos donde los fluidos de trabajo son poco ácidos, se escogen generalmente materiales como latón o bronce y en el caso de los fluidos altamente corrosivos se utiliza, acero inoxidable o mónel [7]. Con respecto a los rangos de presiones de trabajo, éstos pueden abarcar desde pocos psi, aproximadamente $10 \mathrm{psi}$, hasta presiones del orden de los 100.000 psi. Debido a su construcción y funcionamiento, el tubo de Bourdon debe satisfacer unos requerimientos de diseño específicos, siendo todos dependientes de su campo de aplicación. Parámetros como diámetro, materiales, configuración (helicoidal, espiral o forma en C), sección transversal y espesor son los puntos críticos del tubo de Bourdon y deben seleccionarse muy cuidadosamente para garantizar un funcionamiento óptimo del instrumento [1]. Varios autores como Dressler [10] y Austin [11] han llegado a la conclusión que el factor más determinante en cuanto a la sensibilidad, rango de aplicación y precisión de un tubo de Bourdon es el espesor del tubo, debido que es el parámetro que determina qué tanto puede deformarse antes de fallar. La Fig. 3 muestra los distintos espesores de tubo de Bourdon según la presión de trabajo del manómetro. 


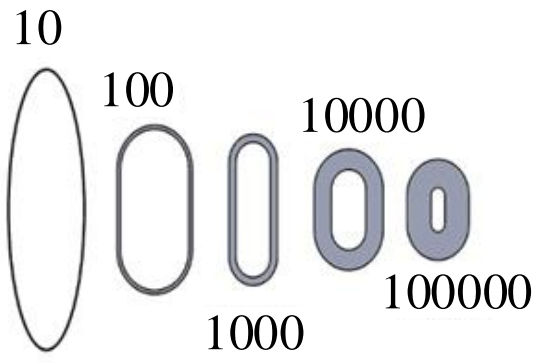

Fig. 3. Ilustración de secciones transversales típicas para rangos de presión en psi.

Se han realizado muchos estudios sobre la deformación del tubo de Bourdon para determinar los esfuerzos y deformaciones a los es sometido para así predecir su trayectoria, pero debido a la complejidad de los cálculos relativos a los cambios de sección transversal por el efecto de la presión y la deflexión producida por el momento con respecto al extremo fijo del manómetro, la mayoría de aproximaciones teóricas tienen errores entre el 4.5 y $4.050 \%$. Esto es debido que generalmente se asume el incremento del radio del tubo de Bourdon con respecto a la presión aplicada como lineal, en contraste con las aproximaciones teóricas no lineales que tienen errores cercanos al 25\% [12]. Por lo anterior, varios autores han concluido que las mejores aproximaciones se dan por medio de métodos de elementos finitos o simulaciones por computadora, como el caso de Bansil [13], quien elaboró un programa de análisis por elementos finitos donde determinaba con gran precisión los esfuerzos y deformaciones de un tubo de Bourdon con sección transversal elíptica plana.

\section{Mecanismos de Cuatro Barras en el Manómetro}

El análisis de cinemática de posición del mecanismo de cuatro barras dentro del manómetro de Bourdon se realiza para hallar el ángulo de salida $\theta_{4}$, el cual indicará la presión en el dial del instrumento. La Fig. 4 muestra un mecanismo clásico de cuatro barras, donde $\mathrm{L}_{1}, \mathrm{~L}_{2}, \mathrm{~L}_{3}$ y $\mathrm{L}_{4}$ son las barras que componen el eslabonamiento de cadena cerrada. El ángulo $\theta_{2}$ representa la entrada del mecanismo, $\theta_{4}$ es el ángulo de salida del mecanismo, y $\gamma$ es el ángulo existente entre el eslabón 2 y 3.

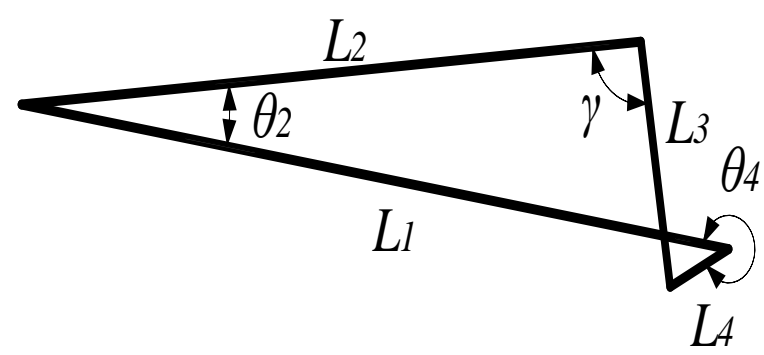

Fig. 4. Mecanismo equivalente de 4 barras en el manómetro.

Se utilizó un manómetro de Bourdon promedio de aplicaciones hidráulicas (agua) con rango de 0 a 60 psi. El análisis inicia estableciendo el ángulo de entrada del mecanismo en cada unidad de presión simulada $\theta_{2}$. Conocidas cada una de las posiciones de la punta del tubo de Bourdon en las presiones de operación y con la ayuda del teorema del coseno, se calcula el ángulo $\theta_{2}$. Debido que las posiciones de la deformación del tubo están tomadas con respecto al origen $(0,0)$ del tubo de Bourdon, se necesita cambiar el punto de referencia para utilizar el teorema del coseno. Para la coordenada X se restan $12.4 \mathrm{~mm}$ y para la coordenada $\mathrm{Y}$ se suman $7.20 \mathrm{~mm}$, hecho esto el nuevo origen se ubica en $(12.40,-7.20)$. Con el nuevo origen determinado se utiliza (1) para hallar la distancia entre dos puntos y determinar la longitud $\mathrm{C}$ del lado del triángulo de la Fig. 5, esta longitud varía con cada presión de trabajo que se simule en el tubo de Bourdon. Las longitudes restantes A y B son de los eslabones $\mathrm{L}_{2}$ y $\mathrm{L}_{1}$, respectivamente, el eslabón $\mathrm{L}_{2}$ se halló en el anexo como la transformación del tubo de Bourdon en un elemento rígido y el eslabón $\mathrm{L}_{3}$ se halló midiendo la pieza directamente del manómetro con un calibrador pie de rey.
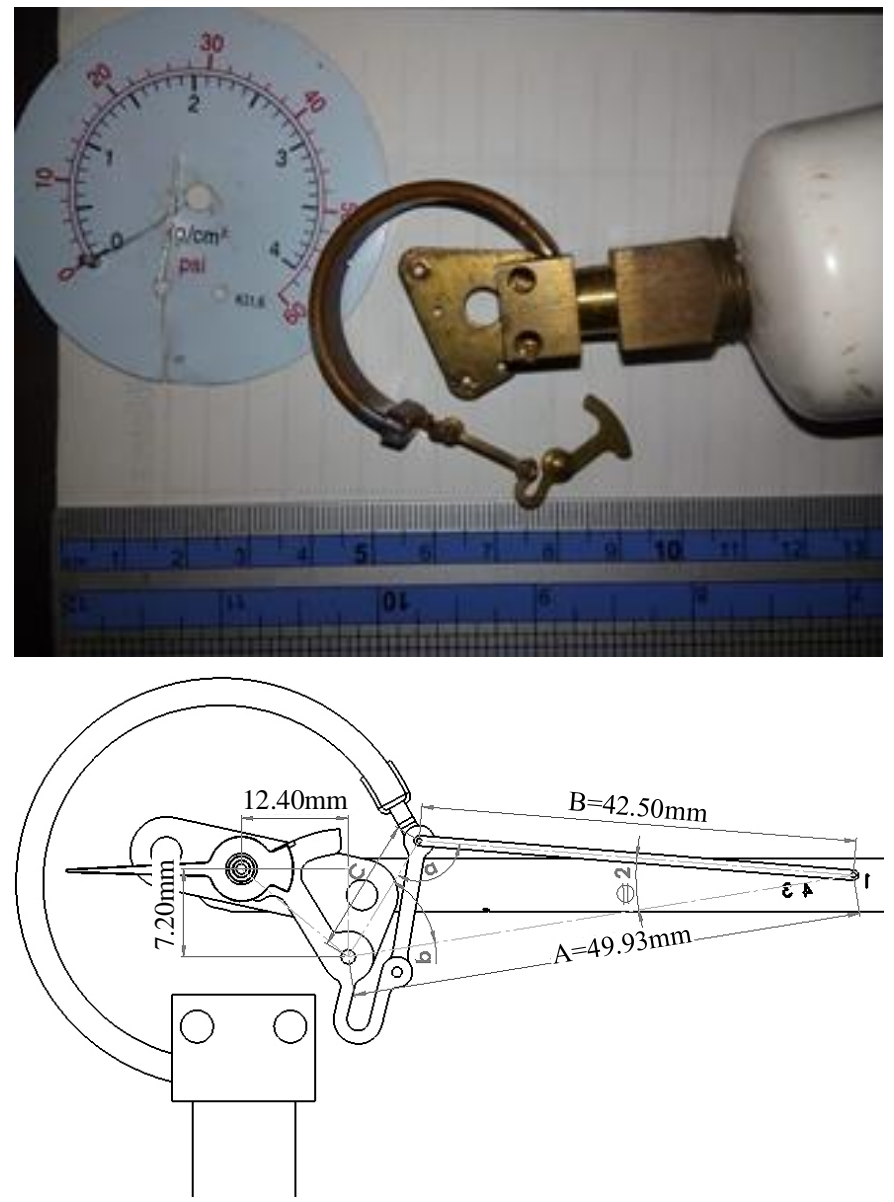

Fig. 5. Aplicación de la Ley del Coseno.

$$
\mathrm{C}=\sqrt{\left(\mathrm{X}_{\mathrm{f}}-\mathrm{X}_{\mathrm{i}}\right)^{2}+\left(\mathrm{Y}_{\mathrm{f}}-\mathrm{Y}_{\mathrm{i}}\right)^{2}}
$$

En la ecuación (1), $X_{\mathrm{i}}$ y $Y_{\mathrm{i}}$ es la coordenada inicial en estado de reposo del mecanismo. Por tanto, $X_{\mathrm{f}} \mathrm{y} \mathrm{Y}_{\mathrm{f}}$ es la coordenada final generada a partir de la presión medida por el tubo de Bourdon. Conocidos los tres lados del triángulo, se utiliza el teorema del coseno de (2) para determinar el ángulo $\theta_{2}$ en cada simulación y teniendo en cuenta que se conocen dos de los tres 
lados del triángulo siendo $\mathrm{A}=49.93 \mathrm{~mm}$ y $\mathrm{B}=42.50 \mathrm{~mm}$. De la (2) se obtiene $\theta_{2}$, como se indica en (3).

$$
\begin{gathered}
C=A^{2}+B^{2}-2 A B \cos \left(\theta_{2}\right) \\
\theta_{2}=\cos ^{-1}\left(C^{2}-A^{2}-B^{2}+2 A B\right)
\end{gathered}
$$

Conocidos todos los ángulos de entrada $\theta_{2}$, con los que opera el manómetro de Bourdon desde 0 hasta 60 psi, se utiliza el análisis de mecanismos de cuatro barras descrito en [14] donde se tiene que el ángulo de salida $\theta_{4}$ está dado por (4) y el ángulo $\gamma$ por (5), respectivamente.

$$
\begin{gathered}
\theta_{4}=2 \tan ^{-1}\left(\frac{\mathrm{L}_{2} \sin \left(\theta_{2}\right)+\mathrm{L}_{3} \sin (\gamma)}{\mathrm{L}_{4}-\mathrm{L}_{1}+\mathrm{L}_{2} \cos \left(\theta_{2}\right)-\mathrm{L}_{3} \cos (\gamma)}\right) \\
\gamma=\cos ^{-1}\left(\frac{\mathrm{L}_{3}^{2}+\mathrm{L}_{4}^{2}-\mathrm{L}_{1}^{2}-\mathrm{L}_{2}^{2}+2 \mathrm{~L}_{1} \mathrm{~L}_{2} \cos \theta_{2}}{2 \mathrm{~L}_{3} \mathrm{~L}_{4}}\right)
\end{gathered}
$$

Después de haber encontrado todos los ángulos de salida $\theta_{4}$, correspondientes a todos los ángulos de entrada $\theta_{2}$, se aplicará interpolación para encontrar las curvas de paso de los engranajes no circulares que regularían las medidas de presión en el dial del instrumento.

\section{ENGRANAJES NO CIRCULARES}

Los engranes no circulares son comúnmente conocidos como generadores de funciones, debido que pueden describir a través de su curva de paso distintas relaciones de transmisión a lo largo de su recorrido, ante una entrada constante de movimiento [6]. Algunos aspectos básicos para la síntesis de las curvas de paso se presentan a continuación.

\section{A. Relación de Transmisión de Engranajes no Circulares}

La razón del cambio infinitesimal del desplazamiento angular del engrane de salida $\mathrm{d} \theta_{\mathrm{o}}$, al cambio infinitesimal del ángulo del engrane de entrada $\mathrm{d} \theta_{\mathrm{i}}$, define la relación de engranajes, g, para el caso de dos engranes planos no circulares, como se presenta en (6). Se considera ángulo de salida $\theta_{\mathrm{o}}$, aquel que está asociado con la rotación del cuerpo conducido, y ángulo de entrada $\theta_{\mathrm{i}}$, aquel asociado con el cuerpo conductor.

$$
g=\frac{d \theta_{0}}{d \theta_{i}}
$$

\section{B. Planeación de Trayectoria}

Una vez se han obtenido los ángulos de entrada y de salida del mecanismo del manómetro de Bourdon, se procede a regular el campo de medida del instrumento usando un método de planeación de trayectoria que dará como resultado la curva de relación de transmisión ideal del sistema. La teoría sobre "Path Planning", expresada en [15], está enfocada al movimiento de un sistema articulado con velocidades y posiciones en el espacio que necesitan conocerse, pero aplicado al caso de estudio, se pueden trabajar las variables en términos de desplazamiento angular y relaciones de transmisión ideales en los rangos de presión del instrumento. Dependiendo de la cantidad de condiciones de frontera que necesite el sistema para delimitar una trayectoria, así será el número de ecuaciones necesarias para solucionarlo. En este caso las condiciones de frontera necesarias para linealizar los rangos de presión son cuatro, la relación de transmisión de entrada $\mathrm{rt}_{\mathrm{i}}$, la relación de transmisión de salida $\mathrm{rt}_{\mathrm{f}}$, el ángulo inicial del rango $\theta_{\mathrm{i}} \mathrm{y}$ el ángulo final del rango $\theta_{\mathrm{f}}$.

Conocidas estas condiciones de frontera, se define la ecuación del "Path Planning" como un polinomio cúbico, definido en (7), compuesto por 4 coeficientes ( $a_{0}, a_{1}, a_{2}$ y a $a_{3}$ ) que satisfagan las condiciones de relación de transmisión y movimiento angular en los puntos iniciales y finales de cada uno de los rangos de presión. Dado que se conocen los parámetros ideales para regular las escalas de presión, sólo es necesario conocer los coeficientes $\mathrm{a}_{\mathrm{i}}$ para generar la trayectoria. En (7) se hace referencia a las posiciones angulares de los rangos de presión, es necesario derivar la ecuación para describir las relaciones de transmisión en los puntos inicial y final, mostradas en la Fig. 6 la cual resume las condiciones de frontera que se presentan en las ecuaciones (8) a (11). La Fig. 6(a) ilustra las condiciones de valores de la relación de engranajes no circulares, mientras que la Fig. 6(b) lo hace para las condiciones de pendiente que garantizan la suavidad del disco entre las diferentes secciones, donde $\mathrm{g}_{\mathrm{w}}$ : relación de engranajes de la sección de trabajo, $\mathrm{g}_{\mathrm{nw}}$ : relación de engranajes de la sección de no trabajo, $\theta_{\mathrm{i}}$ : ángulo del disco no circular de entrada o conductor, $\theta_{\mathrm{f}}$ : ángulo final de la sección working o de trabajo, $\theta_{0}$ : ángulo inicial de la sección working o de trabajo, $\mathrm{g}_{\mathrm{w}}^{\prime}$ : derivada de la relación de engranaje desde la sección de trabajo, $\mathrm{g}_{\mathrm{nw}}^{\prime}$ : derivada de la relación de engranaje desde la sección de no trabajo.

$$
g_{i}(\theta)=a_{0}+a_{1} \theta+a_{2} \theta^{2}+a_{3} \theta^{3}
$$

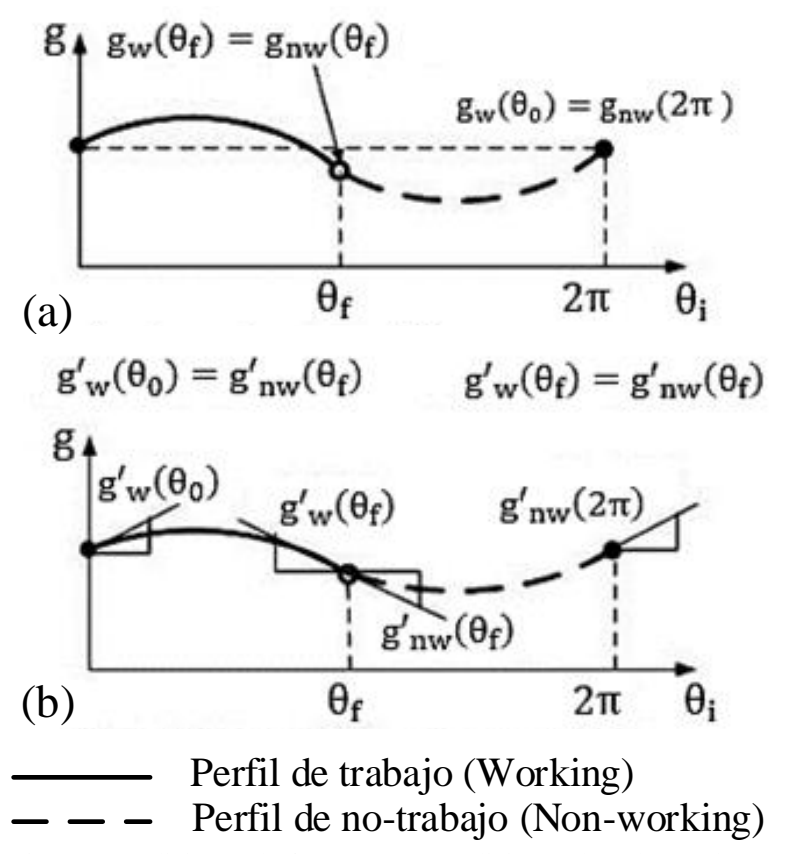

Fig. 6. Condiciones de frontera (a) Condiciones de valores, (b) Condiciones de pendiente. 


$$
\begin{gathered}
g_{w i}\left(t_{0}\right)=g_{w 0} \\
g_{w i}^{\prime}\left(t_{0}\right)=g_{w 0}^{\prime} \\
g_{w i}\left(t_{f}\right)=g_{w f} \\
g_{w i}^{\prime}\left(t_{f}\right)=g_{w f}^{\prime}
\end{gathered}
$$

La ecuación (7) se reemplaza en las condiciones (8) a (11) para generar el sistema de ecuaciones en la sección de trabajo, mostradas en (12), (13), (14) y (15), donde $\theta_{0}$ es el ángulo inicial y $\theta_{\mathrm{f}}$ es el ángulo final, de la sección de trabajo, ángulos ideales para obtener una determinada relación de transmisión que permita cumplir con los desplazamientos requeridos en la indicación del dial del manómetro. Su solución se resume matricialmente en (16), para los valores de los coeficientes $a_{i}$. Se reemplazan los coeficientes en la ecuación principal (7) y se grafican cada uno de los rangos de presión.

$$
\begin{gathered}
g_{0}\left(\theta_{0}\right)=a_{0}+a_{1} \theta_{0}+a_{2} \theta_{0}^{2}+a_{3} \theta_{0}^{3} \\
g_{w 0}^{\prime}\left(\theta_{0}\right)=a_{1}+2 a_{2} \theta_{0}+3 a_{3} \theta_{0}^{2} \\
g_{w f}\left(\theta_{f}\right)=a_{0}+a_{1} \theta_{f}+a_{2} \theta_{f}^{2}+a_{3} \theta_{f}^{3} \\
g_{w f}^{\prime}\left(\theta_{f}\right)=a_{1}+2 a_{2} \theta_{f}+3 a_{3} \theta_{f}^{2} \\
{\left[\begin{array}{l}
a_{0} \\
a_{1} \\
a_{2} \\
a_{3}
\end{array}\right]=\left[\begin{array}{cccc}
1 & \theta_{\mathrm{o}} & \theta_{\mathrm{o}}^{2} & \theta_{\mathrm{o}}^{3} \\
0 & 1 & 2 \theta_{\mathrm{o}} & 3 \theta_{\mathrm{o}}^{2} \\
1 & \theta_{\mathrm{f}} & \theta_{\mathrm{f}}^{2} & \theta_{\mathrm{f}}^{3} \\
0 & 1 & 2 \theta_{\mathrm{f}} & 3 \theta_{\mathrm{f}}^{2}
\end{array}\right]^{-1}\left[\begin{array}{c}
\mathrm{rt}_{\mathrm{o}} \\
0 \\
\mathrm{rt}_{\mathrm{f}} \\
0
\end{array}\right]}
\end{gathered}
$$

De la misma manera, se aplica el procedimiento descrito para la sección "Non working", Fig. 6, o de completitud del disco no circular, donde el valor inicial del ángulo de entrada es $\theta_{\mathrm{f}} \mathrm{y}$ el ángulo final es $2 \pi$.

\section{Perfiles de Paso no Circulares}

Una vez conocida la ecuación que describe la curva de transmisión del instrumento regulado, se genera la curva de paso de los engranajes no circulares. Es posible enlazar la relación de transmisión g con las curvas de paso de los engranajes no circulares a través de los radios de entrada, $r_{i}, y$ de salida, $\mathrm{r}_{\mathrm{o}}$, definidos en (17) y (18), respectivamente, de cada uno de los rangos y la distancia entre centros de los engranajes no circulares, donde cd es la distancia entre centros del par de engranajes no circulares que para el caso de estudio tiene un valor de $13,5 \mathrm{~mm}$.

$$
\begin{aligned}
& r_{i}=c d\left(\frac{g}{1+g}\right) \\
& r_{0}=c d-r_{i}
\end{aligned}
$$

Al desarrollar (7) de relación de transmisión y calcular los radios de cada uno de los engranajes no circulares para los rangos de presión establecidos, se necesita encontrar las coordenadas en el plano donde se encuentran cada uno de estos radios, para esto se emplean las ecuaciones (19) a (22), donde $\mathrm{X}_{\mathrm{o}} \mathrm{y}_{\mathrm{o}}$ son las coordenadas cartesianas de cada uno de los radios que componen el engranaje conducido (o salida), $\mathrm{y}$ $\mathrm{X}_{\mathrm{i}}$ y $\mathrm{Y}_{\mathrm{i}}$ son las coordenadas cartesianas de cada uno de los radios que componen el engranaje conductor (o entrada).

$$
\begin{gathered}
\mathrm{X}_{\mathrm{o}}=\mathrm{r}_{\mathrm{o}} \cos \theta_{\mathrm{o}} \\
\mathrm{Y}_{\mathrm{o}}=\mathrm{r}_{\mathrm{o}} \sin \theta_{\mathrm{o}} \\
\mathrm{X}_{\mathrm{i}}=-\mathrm{r}_{\mathrm{i}} \cos \theta_{\mathrm{i}}+\mathrm{cd} \\
\mathrm{Y}_{\mathrm{i}}=\mathrm{r}_{\mathrm{i}} \sin \theta_{\mathrm{i}}
\end{gathered}
$$

Luego se grafican cada uno de los puntos obtenidos por las ecuaciones anteriores para visualizar las curvas de paso de los engranajes no circulares. Se prosigue con la validación en CAD de los perfiles de paso.

\section{MOdelo CAD DEL MANÓMETRO Y PlaneACión DE TRAYECTORIA}

La Fig 7 muestra una vista de modelo CAD del mecanismo interno y el dial de indicación original, además del detalle en la conexión entre los engranes circulares. Se aprecia que los intervalos de diferencias de 10 psi no son idénticos, presentando la mayor diferencia en el intervalo de 0-10 psi.

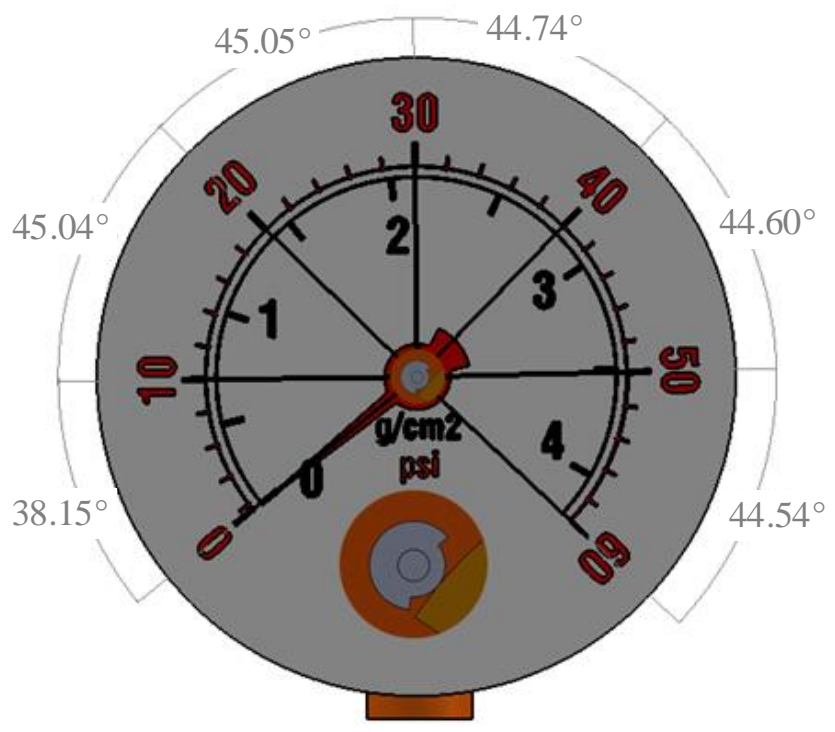

Fig. 7. Dial de indicación original, unidades indicadas.

A través de la simulación por elementos de finitos se determinó la trayectoria definida por la deformación del tubo de Bourdon a lo largo del rango del trabajo, el cual es de 0-60 psi para el instrumento usado como caso de estudio. Esto fue posible construyendo un modelo CAD a escala 1:1 del manómetro de Bourdon utilizando el software de diseño SolidWorks; hecho esto se simuló el mecanismo interno del manómetro en cada una de las presiones de su campo de 
medida. La Fig. 8 muestra el modelo CAD desde una perspectiva tridimensional y un modelo simplificado del mecanismo interno que hace posible la transmisión del movimiento del tubo de Bourdon hacia el dial de indicación del instrumento.

De las simulaciones se obtuvo el perfil de deformación del tubo de Bourdon y con base en éstas se tomaron las coordenadas $\mathrm{X}$ y $\mathrm{Y}$ de la punta del tubo con respecto al centro de la circunferencia del mismo. Definida la trayectoria, se determinó una ecuación que describa este movimiento basándose en la ecuación general de la circunferencia. En la ecuación (23), fue necesario calcular los coeficientes D, E y F para obtener una ecuación en términos de $\mathrm{X}$ y Y. Esto fue posible eligiendo 6 puntos de presión con sus respectivas coordenadas (X, Y) y comprobar que cumpliera lo más cercano posible con la trayectoria marcada por la deformación original. La Tabla I relaciona las coordenadas (X, Y) de los datos usados para determinar los coeficientes D, E y F.

$$
X^{2}+Y^{2}+D x+E y+F=0
$$

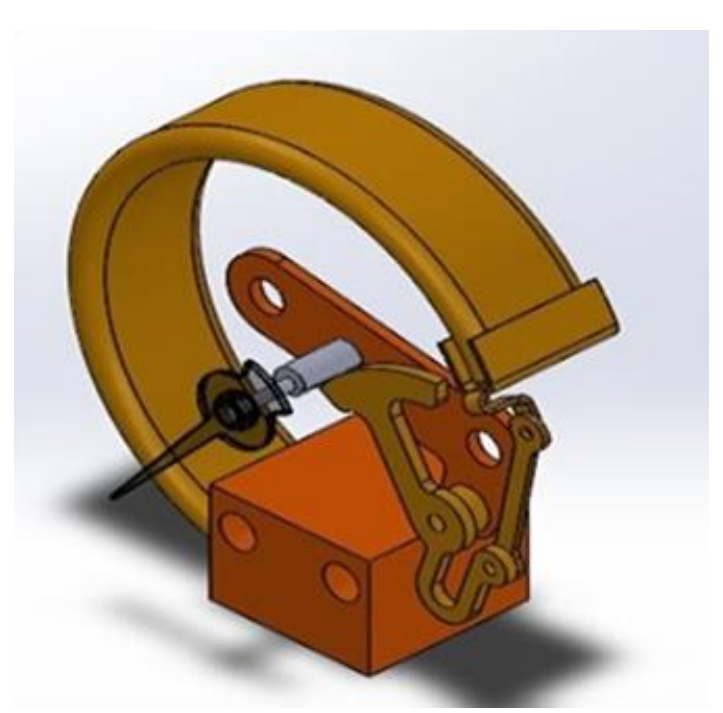

Fig. 8. Modelo CAD del mecanismo interno.

TABLA I

TABLA DE DEFORMACIONES CARTESIANAS

\begin{tabular}{ccc}
\hline \hline Presión[psi] & $X[\mathrm{~mm}]$ & $\mathrm{Y}[\mathrm{mm}]$ \\
\hline 0 & 19.29457 & 3.82514 \\
12 & 19.32123 & 4.17729 \\
24 & 19.34676 & 4.48658 \\
36 & 19.37105 & 4.73876 \\
48 & 19.39673 & 5.03489 \\
60 & 19.42055 & 5.21672 \\
\hline \hline
\end{tabular}

La ecuación (24) muestra los coeficientes que describen la trayectoria circular de las coordenadas de los puntos de presión elegidos, obtenidos mediante programación en Octave. También se determinaron el centro y el radio de esta circunferencia. El centro de esta circunferencia está localizado en $(61.6867 \mathrm{~mm}, 0.76165 \mathrm{~mm})$ con radio $\mathrm{R}=42.5 \mathrm{~mm}$. El radio de la circunferencia es la longitud de un eslabón equivalente, el cual representa el movimiento del tubo de
Bourdon (ángulos de entrada del mecanismo) con el fin de completar un mecanismo de cadena cerrada. Este mecanismo de cadena cerrada permitió determinar los ángulos de salida en la trayectoria recorrida por el eslabón equivalente.

$$
X^{2}+Y^{2}-123.3734 x-1.5233 y+1999.3395=0
$$

En la Fig. 9 se aprecia la inclusión del eslabón equivalente, además de la esquematización mediante un eslabonamiento del mecanismo interno del instrumento mostrado en la Fig. 4. En la Tabla II se presentan las dimensiones del mecanismo equivalente de cuatro barras para el manómetro del caso de estudio.

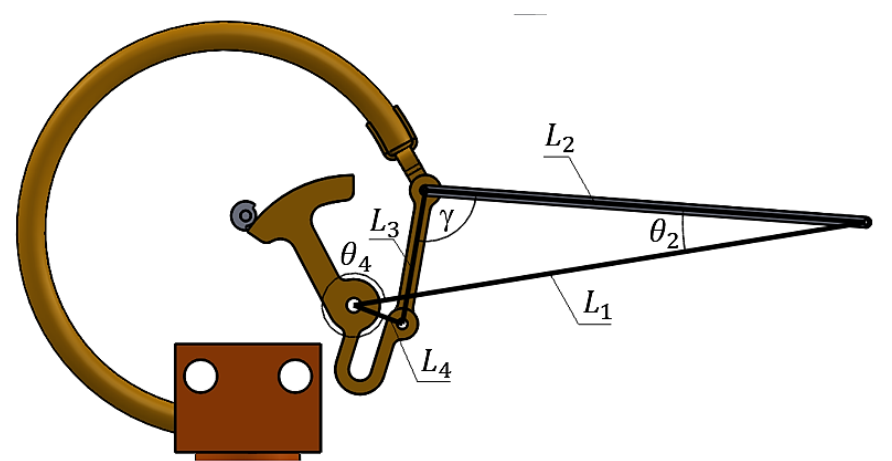

Fig. 9. Mecanismo asociado al Manómetro de Bourdon.

TABLA II

DIMENSIONES DEL ESLABONAMIENTO DE 4 BARRAS.

\begin{tabular}{cc}
\hline \hline Eslabón & Longitud [mm] \\
\hline $\mathrm{L}_{1}$ & 49.92 \\
$\mathrm{~L}_{2}$ & 42.5 \\
$\mathrm{~L}_{3}$ & 13 \\
$\mathrm{~L}_{4}$ & 5 \\
\hline \hline
\end{tabular}

De este mecanismo representado en la Fig. 8 se obtuvieron los ángulos de entrada $\theta_{2}$ del mecanismo, teniendo en cuenta la deformación del tubo de Bourdon en su campo de medida. A su vez, realizando un estudio cinemático directo se halló la fórmula por la cual se obtendría el rango de ángulos de salida $\theta_{4}$ de este mecanismo, los cuales serían los ángulos de entrada para el cálculo de la curva de paso de los engranes no circulares. El ángulo de entrada del mecanismo está dado por (4). La Tabla III relaciona el desplazamiento angular del mecanismo de 4 barras de cadena cerrada por cada 10 psi como presión de entrada.

TABLA III

ÁNGULOS DE ENTRADA Y SALIDA DEL MECANISMO DE 4 BARRAS

\begin{tabular}{ccc}
\hline \hline $\begin{array}{c}\text { Presión de entrada } \\
\text { [psi] }\end{array}$ & $\boldsymbol{\theta}_{\mathbf{2}}\left[^{\circ}\right]$ & $\boldsymbol{\theta}_{\mathbf{4}}\left[{ }^{\circ}\right]$ \\
\hline 0 & 0 & 0 \\
10 & 0.4 & 3.466738 \\
20 & 0.764 & 6.567495 \\
30 & 1.082 & 9.268360 \\
40 & 1.421 & 12.144037 \\
50 & 1.698 & 14.497310 \\
60 & 1.855 & 16.089339 \\
\hline \hline
\end{tabular}


Calculados los ángulos de salida del mecanismo junto con la relación de transmisión (constante) del par de engranes circulares, se comprobó que existe una relación no lineal entre la presión de entrada en el tubo de Bourdon y la presión indicada en el dial del instrumento. Esta relación no lineal es mostrada en la Tabla IV. Se compara la presión medida en el tubo de Bourdon y la indicada en el dial del instrumento. Para compensar esa no linealidad presente en el instrumento, y utilizando los ángulos de salida del mecanismo como ángulos de entrada, se da la síntesis de los engranes no circulares. El desplazamiento angular entre el ángulo de salida del mecanismo equivalente y el dial indicador es la relación de transmisión del engrane no circular, definido en (6).

TABLA IV

TABLA COMPARATIVA DE INDICACIONES

\begin{tabular}{cccc}
\hline \hline $\begin{array}{c}\text { Presión medida } \\
{[\mathrm{psi}]}\end{array}$ & $\begin{array}{c}\text { Ángulo de } \\
\text { entrada }\left[{ }^{\circ}\right]\end{array}$ & $\begin{array}{c}\text { Lectura } \\
\text { indicada [psi] }\end{array}$ & $\begin{array}{c}\text { Lectura regulada } \\
{[\mathrm{psi}]}\end{array}$ \\
\hline 0 & 0.00 & 0.08 & 0 \\
10 & 3.47 & 8.22 & 10 \\
20 & 6.57 & 18.74 & 20 \\
30 & 9.23 & 26.45 & 30 \\
40 & 12.14 & 34.65 & 40 \\
50 & 14.50 & 41.37 & 49.93 \\
60 & 16.09 & 45.91 & 60 \\
\hline \hline
\end{tabular}

Debido a que es necesario indicar la misma presión de entrada en el tubo de Bourdon y en el dial de indicación, se tendrán distintas relaciones de transmisión entre un engrane y otro por lo que consecuentemente se está construyendo y definiendo el paso de cada engrane no circular. En la Tabla V se describe el desarrollo de la planeación de la trayectoria para conseguir la linealización entre la presión de entrada y el dial indicador del instrumento, es decir que la presión medida en el tubo de Bourdon sea la indicada en el dial de instrumento sin elementos externos, por consiguiente, se ha tomado en cuenta rangos de $10 \mathrm{psi}$, con el fin de obtener una transición más suave al momento de construir la curva de paso del engrane circular.

TABLA V

PARÁMETROS DE "PATH PLANNING"

\begin{tabular}{cccc}
\hline \hline $\begin{array}{c}\text { Ángulo de } \\
\text { entrada }\left[{ }^{\circ}\right]\end{array}$ & $\begin{array}{c}\text { Ángulo de } \\
\text { indicación }\left[{ }^{\circ}\right]\end{array}$ & $\begin{array}{c}\text { Rt ideal [sin } \\
\text { dimensión] }\end{array}$ & $\begin{array}{c}\text { Rango de } \\
\text { operación [psi] }\end{array}$ \\
\hline 0 & 0 & 12.5 & 0 \\
3.47 & 45 & 12.98 & $0-10$ \\
6.57 & 90 & 13.7 & $10-20$ \\
9.27 & 135 & 14.57 & $20-30$ \\
12.14 & 180 & 14.82 & $30-40$ \\
14.5 & 225 & 15.52 & $40-50$ \\
16.09 & 270 & 16.78 & $50-60$ \\
\hline \hline
\end{tabular}

Considerando los datos de la Tabla V se ha empleado la fórmula de "Path Planning" con las relaciones de transmisión final e inicial en cada rango de indicación, completando así la matriz descrita en (14) que permite hallar los coeficientes de la fórmula que describe tal trayectoria. De la ecuación (25) se completa cada rango de 10 psi de indicación al igual que en (16). Esta es construida por distintas ecuaciones que juntas describen las distintas relaciones de transmisión que han sido calculadas teniendo en cuenta la separación angular entre cada rango, esto se ha realizado tanto en la sección de trabajo de los engranes no circulares como en la sección de no trabajo, el resultado se ha graficado en la Fig. 10.

$$
g=a_{0}+a_{1}\left(\theta_{f}-\theta_{0}\right)+a_{2}\left(\theta_{f}-\theta_{0}\right)^{2}+a_{3}\left(\theta_{f}-\theta_{0}\right)^{3}
$$

La completitud del disco circular se da básicamente teniendo en cuenta la última relación de transmisión obtenida y la relación de transmisión inicial y el "Path Planning" para completar los 360 grados de un engrane circular regular. Esto ha sido posible usando la matriz descrita en (12).

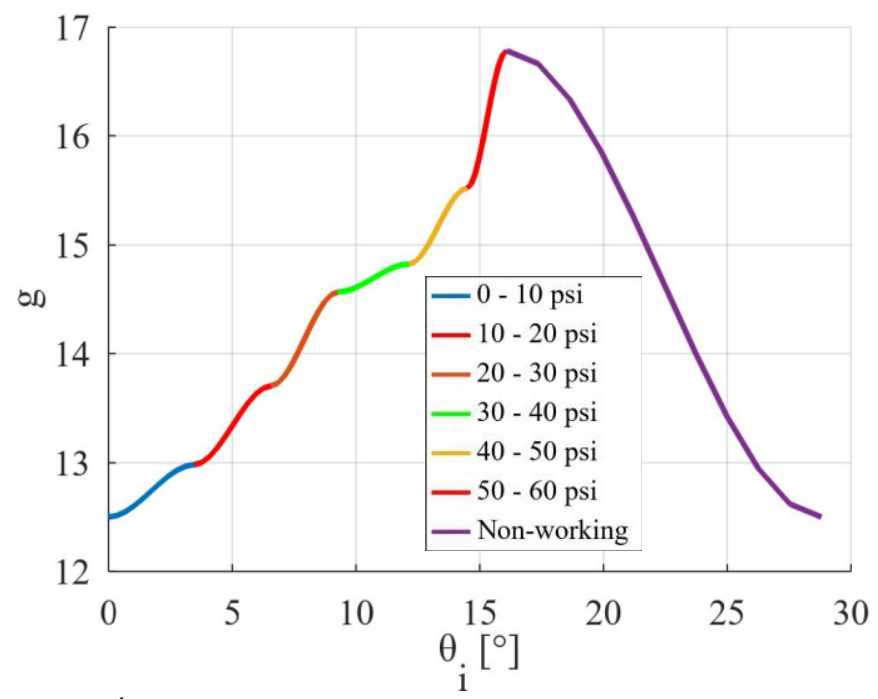

Fig. 10. Ángulo de entrada vs. Relación de transmisión.

\section{SÍNTESIS DE ENGRANAJES NO CIRCULARES}

Una vez se ha obtenido la relación de transmisión a lo largo de la sección de trabajo y de no trabajo, se calcularon los radios del par de engranes teniendo en cuenta (17) a (22) y la distancia de centros establecida. Estos radios obtenidos esbozarán la curva de paso del engrane conductor y el conducido que más tarde se introducirán en el mecanismo del manómetro de Bourdon. Se ha escogido una distancia entre centros de diseño de los engranes no circulares de $13.5 \mathrm{~mm}$ de acuerdo al tamaño del manómetro de referencia, y así calcular los respectivos radios característicos de cada relación de transmisión en cada desplazamiento angular. La Fig. 11 muestra la curva de paso del engrane no circular conducido, el cual es el encargado de la indicación en el dial del instrumento.

Así mismo, la Fig. 12 muestra la curva de paso del engrane no circular conductor que transmite el movimiento proveniente del mecanismo interno del instrumento. Finalmente se presenta la Fig. 13 integrando los perfiles de los engranes no circulares generados en Octave. La Fig. 14 muestra cómo el engrane no circular logra linealizar la relación entre la presión medida por el tubo de Bourdon y la indicada en el dial del instrumento; a la vez que se compara 
con los resultados de indicación del instrumento original que emplea engranes circulares regulares.

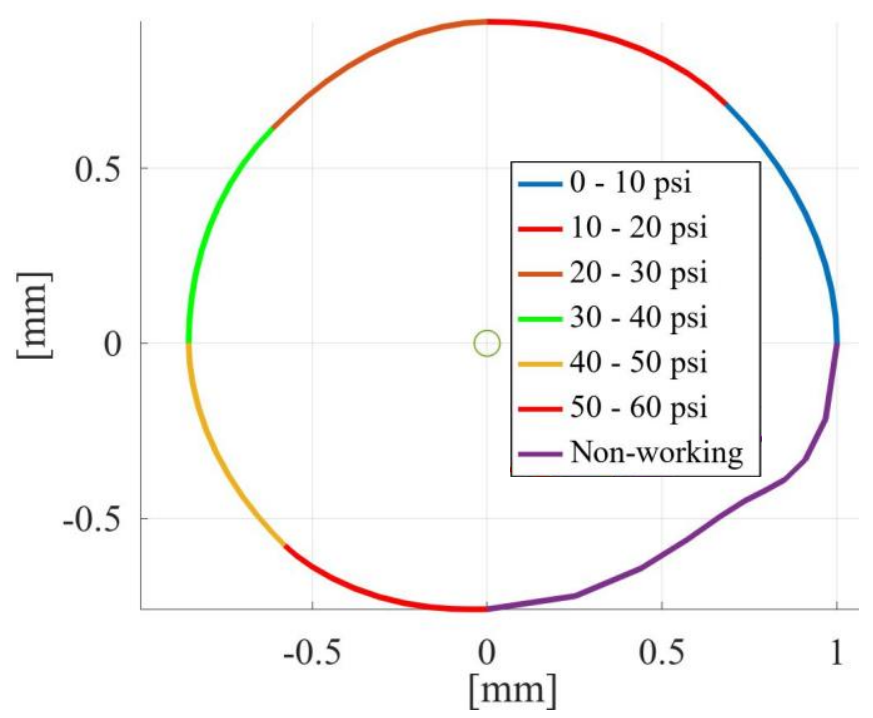

Fig. 11. Curva de paso del piñón (engrane conducido).

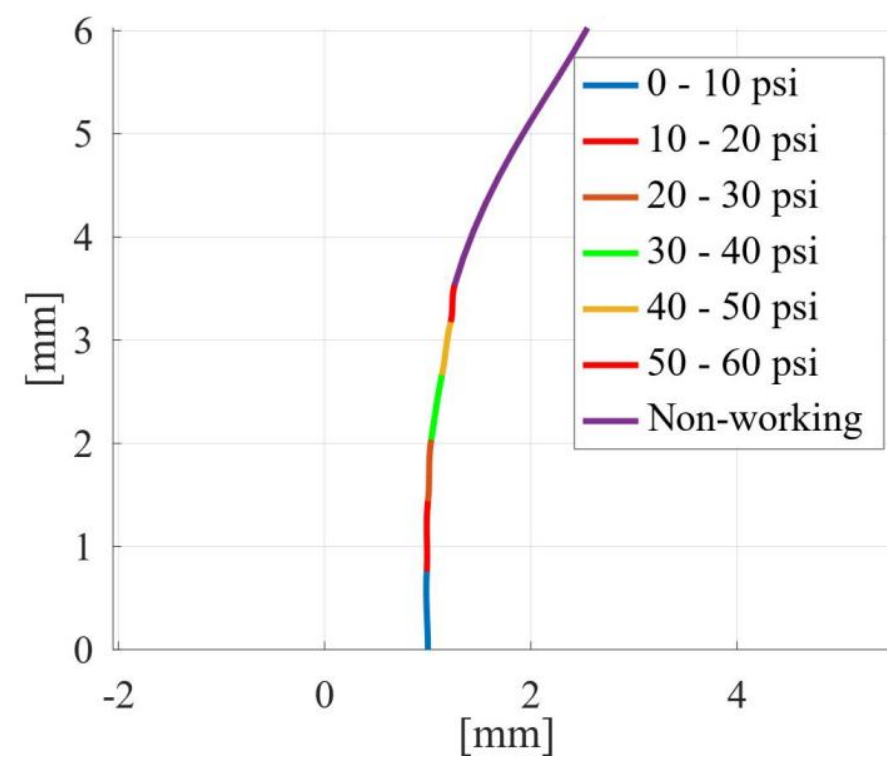

Fig. 12. Curva de paso del engrane conductor.

\section{VALIDACIÓN GRÁFICA}

Definida la curva de paso del par de engranes no circulares y exportando los radios característicos de éstos para generar un modelo CAD. Este modelo fue introducido en el mecanismo del manómetro de Bourdon para su validación y poder correlacionar los datos obtenidos en la simulación en Octave y el software usado para genera el CAD, en este caso, SolidWorks. En la Fig. 15 se aprecia el cambio en las porciones angulares correspondientes a los intervalos de 10 psi. Al compararse con la disposición original de la Fig. 7, se puede deducir que la porción angular de la medición ha cambiado, pasando de $262.12^{\circ}$ a casi $270^{\circ}\left(269.35^{\circ}\right)$.

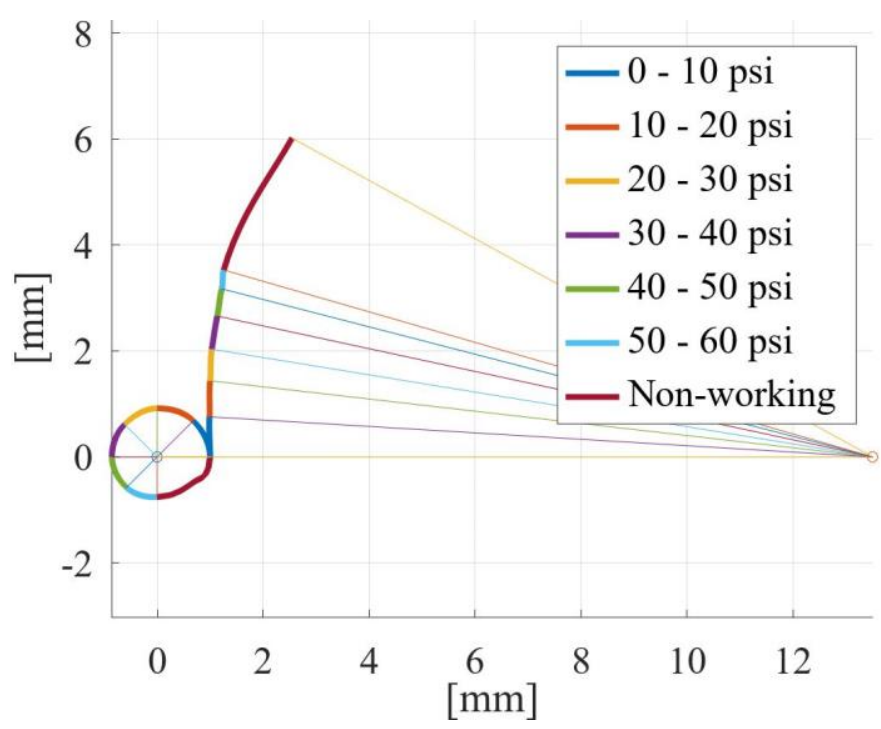

Fig. 13. Integración de los perfiles de paso de los engranajes no circulares.

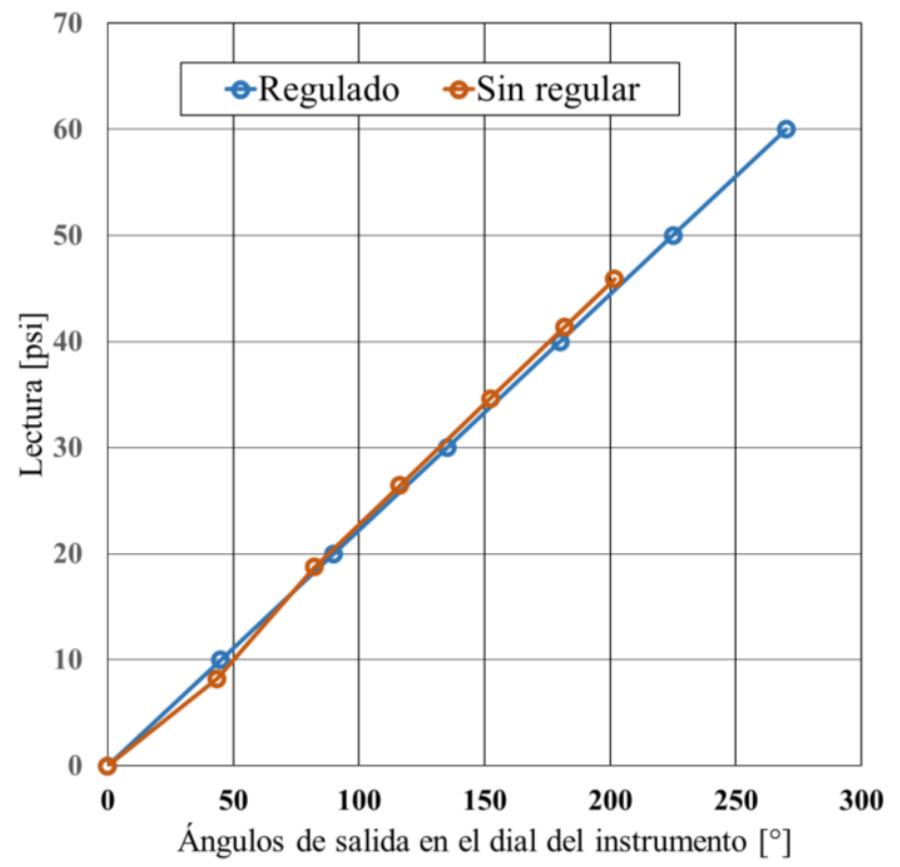

Fig. 14. Rango de trabajo linealizado a $45^{\circ}$.

El nuevo valor del alcance, se ha distribuido en 6 regiones que van de $44.37^{\circ}$ como valor mínimo, a $45.58^{\circ}$ como valor máximo, para una diferencia máxima de $1.21^{\circ}$. Antes de la linealización, para la disposición de la Fig. 7 se tendría una diferencia entre valores máximo y mínimo de $6.9^{\circ}$, resultado de la diferencia de $45.05^{\circ}$ como valor máximo y $38.15^{\circ}$ de valor mínimo. Lo anterior muestra una mejora en el rango de media, especialmente cerca al límite inferior de medida. La Fig. 15 también presenta un acercamiento del contacto de las curvas de paso del engranaje conducido con la porción conductora. De otra parte, la Fig. 16 muestra las porciones angulares del disco conducido completo, con la forma del disco conductor y su porción de trabajo. 


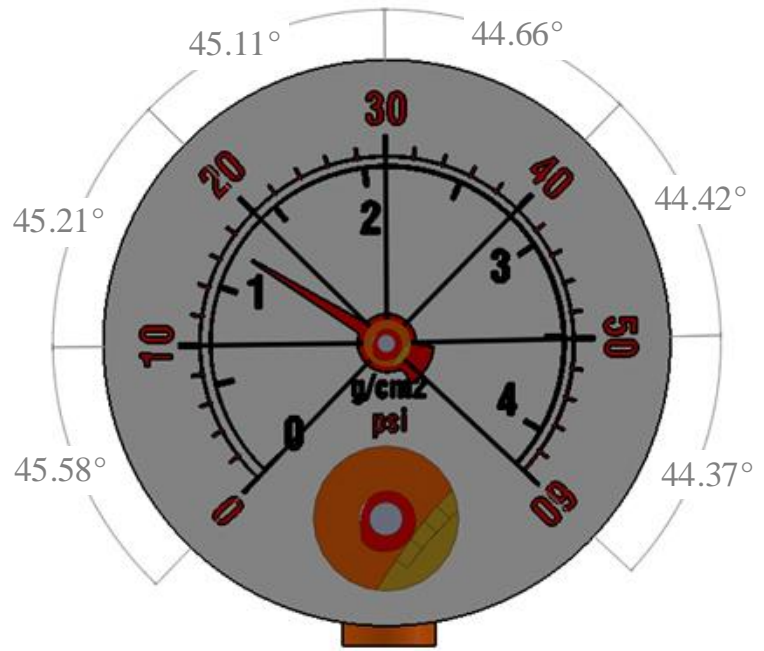

Fig. 15. Dial de indicación linealizado, unidades indicadas.

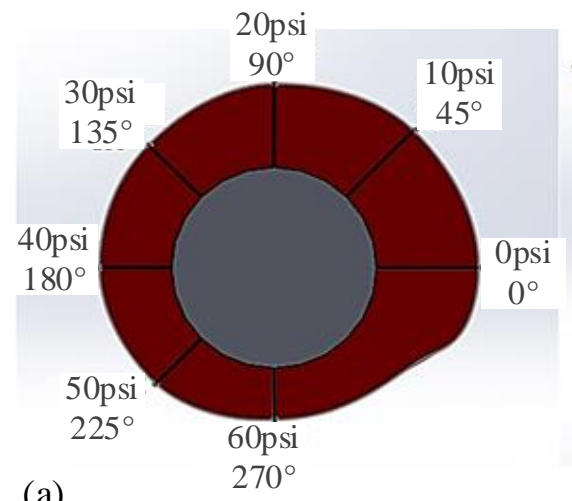

(a)

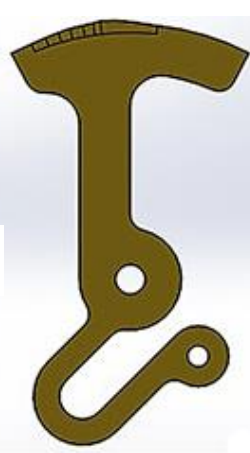

(b)

Fig. 16. Modelo CAD de los engranajes no circulares. a) Piñón conectado a aguja indicadora, b) segmento conductor conectado a eslabonamiento de 4 barras.

\section{CONCLUSIÓN}

Fue reportada la integración de engranes no circulares en el mecanismo de indicación del manómetro de Bourdon. Se ha obtenido como resultado una relación lineal entre la presión medida por el tubo de Bourdon y la presión indicada en el dial del instrumento. Esto ha sido posible mediante la simulación de la trayectoria del tubo de Bourdon con la que fue posible modelar un eslabón equivalente que permitiera completar un mecanismo de 4 barras de cadena cerrada. A este mecanismo de 4 barras de cadena cerrada se le realizó un análisis cinemático, estableciendo los ángulos de entrada y de salida del mecanismo para obtener la relación de transmisión del par de engranes no circulares, calculando la curva de paso de los engranes no circulares a través de un código de programación en Octave, con el que se obtuvo la relación de transmisión de los engranes no circulares.

La generación de la curva de paso precedió la respectiva validación de datos, realizando un modelo CAD de los perfiles de paso no circulares e insertándolos en el modelo CAD original del manómetro de Bourdon para analizar su efecto, además de corroborar que efectivamente, los datos obtenidos en la simulación de Octave corresponden a la realidad del modelo CAD. La principal consecuencia de esta relación 1:1 es conseguir que la escala de medición tenga una resolución uniforme en todo el campo de medida, además de que el instrumento sea capaz de indicar una presión desde el instante en que el tubo de Bourdon sea deformado por la presión de entrada, sin necesidad de emplear elementos externos que consigan impulsar el mecanismo. La principal contribución de este trabajo fue contrarrestar la no linealidad en la salido de la aguja indicadora del manómetro Bourdon mediante el uso de elementos mecánicos, sin necesidad de algoritmos de trasducción. Como trabajo futuro derivada de esta aplicación, se plantea el aumento del tamaño del disco conductor a un mayor diámetro que facilite la manufactura de los elementos no circulares.

\section{AGRADECIMIENTOS}

Los autores agradecen el apoyo brindado por la Vicerrectoría de Investigaciones, Extensión y Proyección Social de la Universidad del Atlántico.

\section{REFERENCIAS}

[1] P. Exline, "Bourdon tube deflection characteristics," Transaction of the ASME, Journal of Basic Engineering, vol. 82, no. 4, pp. 887-892, Dec. 1960. Accessed on: 06, 25, 2017, DOI: 10.1115/1.3662791, [Online].

[2] L. Beiner, "Optimal design of Bourdon gage mechanisms," Transactions of the ASME, Journal of Mechanisms, Transmissions, and Automation in Design, vol. 109, no. 4, pp. 524-527, Dec. 1987. Accesed on: 06, 25, 2017, DOI: 10.1115/1.3258831, [Online]

[3] B. Roth and F. Freudenstein, "Discussion: "Geared five-bar motion: part 1-gear ratio minus one"," Transactions of the ASME, Journal of Engineering for Industry, vol. 31, no. 2, pp. 360-361, Jun. 1963. Accessed on 06, 25, 2017, DOI: 10.1115/1.3629629.

[4] D. B. Dooner, "Fundamental principles of toothed bodies in mesh," in Kinematic Geometry of Gearing, 2nd ed., Chichester, United Kingdom: Wiley, 2012, pp. 33-39.

[5] D. Dooner, "Function generation utilizing an eight-link mechanism and optimized non-circular gear elements with application to automotive steering," Proceedings of the Institution of Mechanical Engineers, Part C: Journal of Mechanical Engineering Science, vol. 215, no. 7, pp. 847-857, Jul. 2001. DOI: 10.1243/0954406011524090, [Online].

[6] D. Dooner, "Use of noncircular gears to reduce torque and speed fluctuations in rotating shafts," Transactions of the ASME, Journal of Mechanical Design, vol. 119, no. 2, pp. 299-306, Jun. 1997. Accessed on 06, 25, 2017, DOI: 10.1115/1.2826251, [Online].

[7] F. Machuca-Martínez y S.Reif-Acheman, "Eugène Bourdon y la evolución del manómetro," Revista Brasileira de Ensino de Física, vol. 32, no. 1, Mar. 2010.

[8] S. B. Reddy, "Instrumentation Tools," [Online]. Available: https://instrumentationtools.com/types-of-bourdon-tube/, Accessed on: Oct. 24, 2018.

[9] WIKA Handbook Pressure and Temperature Measurement, WIKA Instrument Corporation, Lawrenceville, GA, USA, 2008.

[10] R. Dressler, "Elastic shell-theory formulation for Bourdon tubes," Transactions of the ASME, Journal of Basic Engineering, vol. 87, no. 4, pp. 1072-1077, Dec. 1965. Accessed on: 06, 25, 2017, DOI: 10.1115/1.3650809, [Online].

[11] A. Austin and J. Swannell, "Stresses in a pipe bend of oval crosssection and varying wall thickness loaded by internal pressure," International Journal of Pressure Vessels and Piping, vol. 7, no. 3, pp. 167-182, May 1979. Accessed on: 06, 25, 2017, DOI: 10.1016/03080161(80)90019-8, [Online].

[12] G. Smith, "A study of Bourdon tube deflection using numerical analysis solution," Oklahoma State University of Agriculture and Applied Science, Stillwater, Oklahoma, May. 1952.

[13] M. Bansil and F. Abdullah, "Finite element modelling of Bourdon tubes used in pressure and temperature gauges," Transactions of the Institute of Measurement and Control, vol. 7, no 4, pp. 203-208, Jul. 
1985. Accessed on: 06, 25, 2017, DOI: $10.1177 / 014233128500700404$. [Online].

[14] J. Shigley, Teoría de Máquinas y Mecanismos. Mexico D.F., Mexico: McGraw Hill, 1980, pp. 29-73.

[15] R. N. Jazar, Theory of Applied Robotics, New York, NY, USA: Springer, 2007, pp. 569-606.

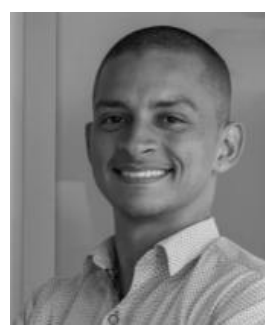

Andrés F. Manotas nacido en Barranquilla, Colombia el 09 de noviembre de 1994. Ingeniero Mecánico de la Universidad del Atlántico del programa de Ingeniería Mecánica. Énfasis en diseño de máquinas y elementos para mecanizado, manufactura aditiva, electrónica y simulación por elementos finitos. Realizó pasantía con la empresa minera Relianz en 2015, luego trabajó como dibujante técnico e ingeniero de obras de 2016 a 2018. Actualmente trabaja en el departamento de diseño de la compañía Energía Solar SA como profesional en especificaciones técnicas.

ORCID: https://orcid.org/0000-0002-8901-5684

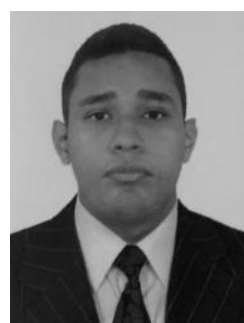

Javier E. Silva-Gutiérrez nacido en Barranquilla, Colombia el 28 de enero de 1994. Ingeniero Mecánico de la Universidad del Atlántico (Puerto Colombia, Colombia) tomando grado en el año 2019. Actualmente se desempeña como investigador asistente en la Universidad del Atlántico. Entre los intereses investigativos se encuentra el diseño de máquinas, instrumentación industrial y control de procesos.

ORCID: https://orcid.org/0000-0002-9351-5876

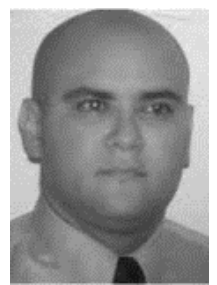

Javier Roldán Mckinley es Ingeniero Mecánico de la Universidad del Atlántico (Puerto Colombia, Colombia) en el año 2001. Obtuvo grado de Doctor en Ingeniería Mecánica en la Universidad de la Florida (Gainesville, FL-USA) en el año 2007. Actualmente trabaja como profesor asociado del Programa de Ingeniería Mecánica de la Universidad del Atlántico (Puerto Colombia, Colombia). Es integrante activo del grupo de investigación DIMER.

ORCID: https://orcid.org/0000-0003-1444-6460 